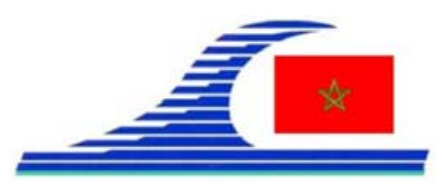

Conférence Méditerranéenne Côtière et Maritime

EDITION 2, TANGER, MAROC (2011)

Coastal and Maritime Mediterranean Conference

Disponible en ligne - http://www.paralia.fr - Available online

\title{
Dynamique tourbillonnaire 2D dans un champ de houle
}

\author{
Adrien POUPARDIN ${ }^{1}$, Gaële PERRET ${ }^{1}$, Jérôme BROSSARD ${ }^{1}$
}

1. Laboratoire Ondes et Milieux Complexes, FRE/CNRS 3102 et Université du Havre, Bâtiment COREVA, 53 rue de Prony, BP 540, 76058 Le Havre Cedex, France. adrien.poupardin@etu.univ-lehavre.fr

\section{Résumé :}

L'interaction des vagues et du courant avec des structures immergées en zone côtière génère des filaments tourbillonnaires qui perturbent l'environnement marin proche. Une étude expérimentale de ces tourbillons est réalisée en considérant une plaque mince horizontale, utilisée comme générateur de tourbillons, immergée dans un canal à houle. La Vélocimétrie par Images de Particules (PIV) et la Stéréo Vidéographie sont utilisées pour caractériser les tourbillons. La PIV montre que la dynamique des tourbillons impacte considérablement l'écoulement autour de la plaque. De plus, les tourbillons, en aval de la plaque, atteignent le fond du canal et pourraient générer de l'affouillement dans une configuration réelle. Grâce aux techniques utilisées, nous pouvons obtenir des informations sur la formation des tourbillons, leur évolution, et leur destruction.

\section{Mots-clés :}

Dynamique tourbillonnaire - Interactions houle/structures - Impacts environnementaux

\section{Introduction}

Avec le développement des énergies renouvelables, les structures immergées, comme les éoliennes offshores ou les hydroliennes, sont amenées à être installées sur le littoral. Cependant, l'interaction entre ces structures et la houle génère des filaments tourbillonnaires qui perturbent l'environnement marin. Ces filaments peuvent atteindre les fonds marins, la surface libre et éventuellement d'autres structures voisines et amoindrir leurs performances. Lorsque les tourbillons atteignent les fonds marins des phénomènes d'affouillement sont observés (SUMER et al., 2001). De plus, les tourbillons induisent des efforts de portance qui peuvent être dimensionnants pour la structure. La structure que nous utilisons est une plaque mince horizontale, utilisée comme générateur de tourbillons. L'étude se concentre alors sur la dynamique des tourbillons sous l'effet de la houle. Les tourbillons sont générés en bord de plaque et se déstabilisent sous l'effet d'instabilités tridimensionnelles. L'objectif de l'étude est de caractériser la dynamique tourbillonnaire en considérant l'intensité, la circulation, la durée de vie et la déstabilisation des tourbillons dans cette configuration idéale. Des théories potentielles (PARAPATANICH, 1984 ; MASSEL, 1983 ; CARTER, 2005) ont été utilisées pour étudier la surface libre et les coefficients de transmission et de réflexion dans cette configuration. Mais ces théories ne peuvent pas être utilisées pour 
La connaissance de la Mer :

un vecteur du développement durable en Méditerranée

décrire correctement l'écoulement autour de la plaque car ce sont des théories potentielles. Nous utilisons donc une approche expérimentale pour comprendre l'évolution 2D des tourbillons.

\section{Dispositif expérimental}

Nous utilisons la PIV (figure 1.a) et la Stéréo Vidéographie (figure 1.b) pour caractériser la dynamique tourbillonnaire autour de la plaque immergée. Nous utilisons un canal à houle pour générer une houle monochromatique à une fréquence de $1 \mathrm{~Hz}$ et une amplitude de $1,1 \mathrm{~cm}$. La plaque immergée a une longueur de $0,25 \mathrm{~m}$ et est immergée à $7 \mathrm{~cm}$ de la surface libre. La hauteur d'eau est 0,20 $\mathrm{m}$. Le rapport longueur de plaque/longueur d'onde est donc 0,21 et le rapport d'immersion 0,35. Dans ces conditions le coefficient de réflexion de la plaque est de $25 \%$.

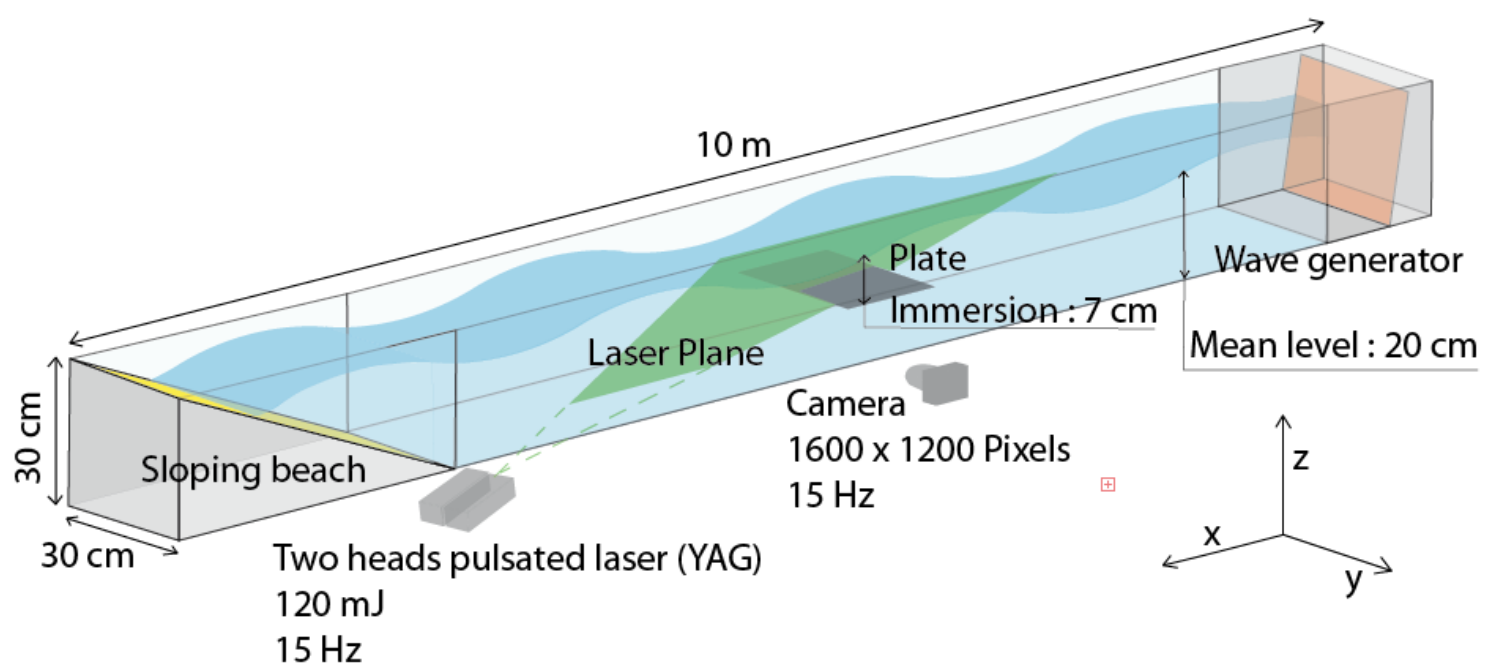

Figure 1. Dispositif expérimental.

\section{Résultats expérimentaux}

Grâce à la PIV, nous suivons l'évolution des tourbillons, depuis leur formation jusqu'à leur destruction. Des paires de tourbillons de signes opposés se forment à chaque période de houle aux deux extrémités de la plaque immergée. Nous déterminons les trajectoires, les durées de vie, les intensités et les circulations moyennes de ces tourbillons. Le champ de vitesse moyen (Figure 2), moyenné sur une période de houle, met en évidence deux cellules de recirculation, sous la plaque, générées par l'advection des paires de tourbillons. En aval, les paires de tourbillons atteignent le fond du canal et créent de l'affouillement. 


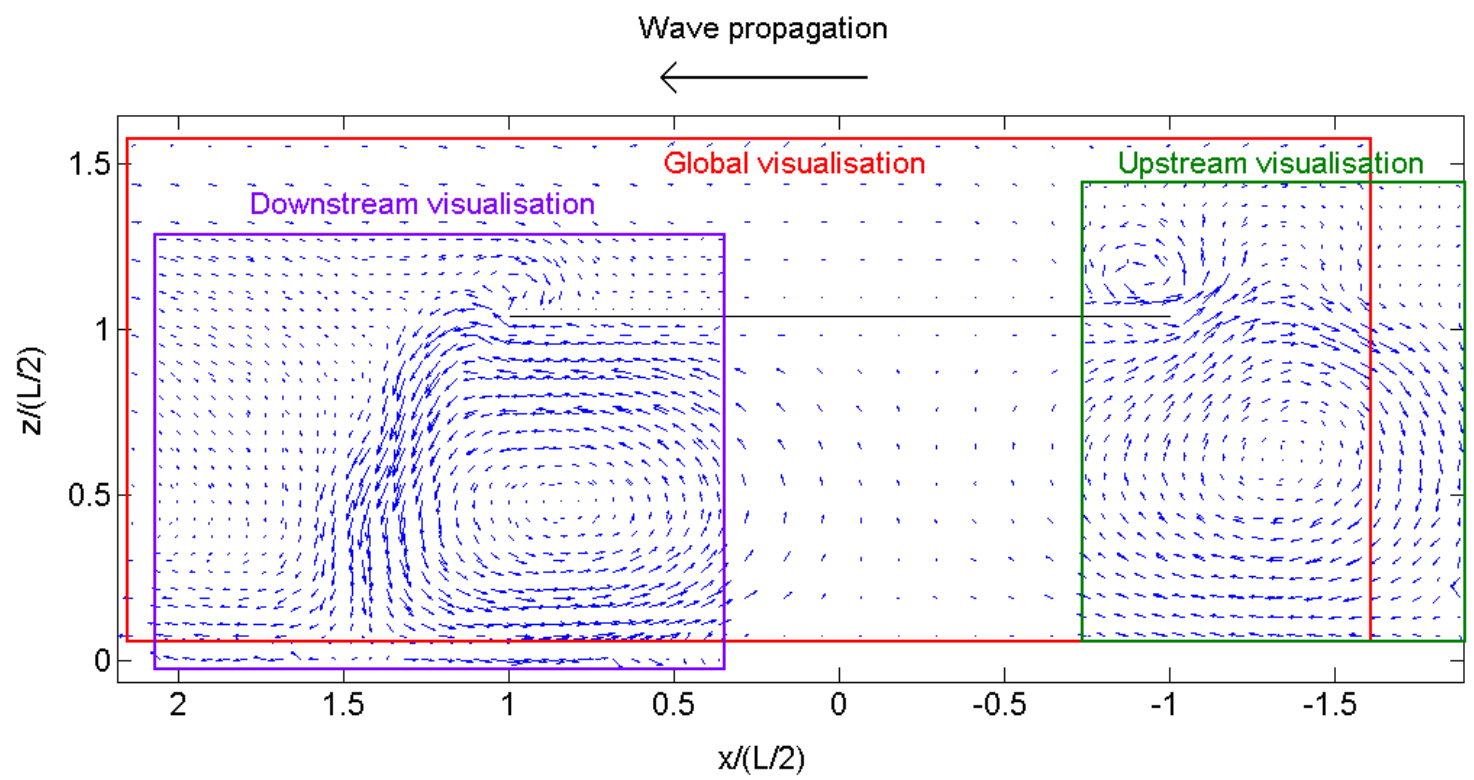

Figure 2. Champ de vitesse moyen.

Ce champ de vitesse moyen est totalement différent du champ de vitesse prédit par les théories potentielles où l'écoulement est considéré comme oscillant sous la plaque (GRAW, 1993 ; CARTER, 2005).

L'interaction mutuelle des tourbillons conduit à leur déstabilisation puis à leur destruction. Ces phénomènes sont fortement tridimensionnels et doivent être étudiés par des méthodes adaptées. La stéréo Vidéographie permet de reconstituer les tourbillons en trois dimensions et d'étudier leurs modes de déformation.

\section{Conclusion et perspectives}

Nous avons prouvé que la dynamique tourbillonnaire influençait fortement l'écoulement autour de la plaque. L'environnement se trouve perturbé par l'implantation de structures dans la houle.

L'analyse 2D de la dynamique tourbillonnaire présentée ici sera poursuivie par une analyse 3D à l'aide d'un système de stéréo-vidéographie afin de connaître les paramètres géométriques et de houle qui conduisent à une forte déstabilisation des tourbillons.

\section{Remerciements}

Ce travail est partiellement financé par la Région Haute Normandie.

\section{Références bibliographiques}

CARTER R.W. (2005). Wave energy converters and a submerged horizontal plate. Thesis of University of Hawaii, Ocean and resources Engineering, 251 p. 
La connaissance de la Mer :

un vecteur du développement durable en Méditerranée

GRAW K.-U. (1993). The submerged plate wave energy converter - A new type of wave energy device, ODEC, Mumoran, Hokkaido, Japan, pp 1-4.

MASSEL S.-R. (1983). Harmonic generation by waves propagating over a submerged plate. Coastal Engineering 7, pp 357-380. doi:10.1016/0378-3839(83)90004-2

PARAPATANICH M. (1984). Maximum and zero reflection from submerged plate, J. of Waterway Port Coastal and Ocean Eng., ASCE, 110(2), pp 171-181. doi:10.1061/(ASCE)0733-950X(1984)110:2(171)

SUMER B.M., WHITEHOUSE R.J.S., TORUM A. (2001). Scour around coastal structures: a summary of recent research. Coastal Engineering 44, pp 153-190. doi:10.1016/S0378-3839(01)00024-2 